\title{
USO Y APLICACIÓN DE HERRAMIENTAS DEL MODELO DE PRODUCCIÓN TOYOTA: UNA REVISIÓN DE LITERATURA
}

\author{
Dairo Steven Muñoz Pinzón', Wilfrido Javier Arteaga Sarmiento², Diana Carolina Villamil Sandoval ${ }^{3}$
}

${ }^{1}$ Ingeniero Industrial. Docente Tiempo Completo. Fundación Universitaria Agraria de Colombia. BogotáColombia.munoz.dairo@uniagraria.edu.co

${ }^{2}$ Magister en gestión de la información y la documentación. Docente Tiempo Completo. Universidad Militar Nueva Granada. Bogotá-Colombia. wilfrido.arteaga@unimilitar.edu.co

${ }^{3}$ Magister en ingeniería con énfasis en sistemas de calidad y productividad. Docente Tiempo Completo. Universidad Militar Nueva Granada.Bogotá-Colombia. diana.villamil@unimilitar.edu.co

\section{RESUMEN}

El presente trabajo muestra una revisión de literatura sobre el uso y aplicación de las diferentes herramientas del modelo Toyota en la industria en los últimos cinco años, haciendo énfasis en las herramientas más empleadas y las variables más representativas de medición de los sistemas productivos. El propósito es conocer la forma en que diversos autores emplean las herramientas de producción ajustada para la mejora de los sistemas productivos y a su vez conocer las principales barreras existentes para su aplicación en las pequeñas y medianas empresas (PYMES) de manufactura. En la revisión de literatura se hizo énfasis en aquellas investigaciones en las que se aplicó alguna de las herramientas Lean involucradas en el estudio. Como resultado del trabajo, se tiene un análisis conceptual respecto a las herramientas Lean y las principales variables de estudio involucradas de acuerdo con la literatura.Como principal conclusión, las herramientas Leandan cumplimiento a los requerimientos de la industria contemporánea, permiten solucionar problemas asociados con análisis de valor, el orden y aseo, la flexibilidad y los impactos ambientales.

Palabras clave: Modelo de producción Toyota; Herramientas Lean; Barreras de implementación; Variables del sistema de producción.

Recibido:25 de septiembre de 2018. Aceptado:28 de Noviembre de 2018

Received: September 25th, $2018 . \quad$ Accepted: November28th, 2018

\section{USE AND APPLICATION OF TOYOTA PRODUCTION MODEL TOOLS: A REVIEW OF LITERATURE}

\begin{abstract}
The present work shows a literature review on the use and application of the different tools of the Toyota model in the industry in the last five years, emphasized in the most used tools and the most representative variables for the improvement of productive systems. The purpose is to know how different authors use the Lean tools for the improvement of the productive systems and at the same time to know the main barriers existing for their application in the small and medium enterprises (SMEs). For the literature review, it was emphasized in those investigations in which some of the Lean tools involved in the study were applied. As a result of the work, we have a conceptual analysis regarding the Lean tools and the main study variables involved according to the literature. As the main conclusion, the Lean tools comply with the requirements of the contemporary industry, allow to solve problems associated with value analysis, order and cleanliness, flexibility and environmental impacts.
\end{abstract}

Keywords: Toyota Production System; Lean Tools; Implementation barriers; Production Systems Variables.

Cómo citar este artículo: D. S. Muñoz Pinzón, W. J. Arteaga Sarmiento, D. C. Villamil Sandoval, "Uso y aplicación de herramientas del modelo de producción Toyota: una revisión de literatura”, Revista Politécnica, vol. 14, no. 27 pp.80-92, 2018. https://doi.org/10.33571/rpolitec.v14n27a8 


\section{INTRODUCCIÓN}

Debido a la globalización y las tendencias del desarrollo tecnológico, las empresas se someten a un entorno altamente competitivo, donde el cliente está bien informado y posee un alto grado de negociación. Las empresas se ven obligadas a mejorar sus sistemas productivos e incluso la organización completa para poder satisfacer las necesidades del cliente. Cada empresa tiene una creciente necesidad de mejorar la calidad, el rendimiento y la voz de la satisfacción del cliente[1] abordando los problemas existentes en los procesos de fabricación [2]. Las empresas con frecuencia emplean diferentes técnicas y herramientas para optimizar la productividad en sus sistemas de fabricación buscando la ventaja competitiva [3], esto entregando los productos adecuados en el lugar correcto y cumpliendo con la entrega a tiempo [4]. Los sistemas de producción existentes a menudo se basan en la filosofía de mejora continua de la gestión Lean[5]para mantener su competitividad frente a las demás empresas, esto buscando un incremento en la productividad delos sistemas de fabricación [6].

El primero en el estudio de LeanManufacturing (LM) fue [7], quien presentó el Modelo de Producción Toyota (TPS) y explicó los principios de Lean para enfocar los esfuerzos de producción de automotores. LM ha sido un aporte importante para la solución de necesidades de la industria, es uno de los métodos utilizados por los fabricantes en todo el mundo para mejorar su competitividad [8]. El propósito principal de Leanes promover la cultura de mejora continua dentro de una empresa [9]. La administración bajo la filosofía Lean incluye un conjunto de herramientas que permiten a las compañías recibir fuertes beneficios cuando se implementan adecuadamente [10].

Existe un gran número de herramientas como lo son el Kaizen, las5s, el Heijunka, el Kanban, el SMED (Cambio de Matriz en un Digito de Minuto), elTPM (Mantenimiento Productivo Total), el Jidoka, el VSM (Mapa de flujo de valor), el Andon (Control Visual), los KPI's (Indicadores Clave de Desempeño), el SixSigma y PokaYoke. Dichas herramientas son de fácil aplicación e involucran a toda la organización, permiten empoderar a los colaboradores y hacer visibles todos los resultados de su trabajo [10]. Las herramientas Lean han contribuido en la mejora de los sistemas productivos en las empresas fabricantes, cumpliendo con los objetivos organizacionales contemporáneos de rentabilidad, eficiencia, receptividad, calidad y atención al cliente [11].

En la literatura es posible encontrar un gran número de teorías y aplicaciones asociadas a LM, en donde, se evidencia la importancia de las herramientas y su impacto en la industria contemporánea. Por lo que, el propósito del presente estudio es conocer la aplicación de las herramientas Lean e identificar las variables más estudiadas en los sistemas productivos. Incluido a este propósito, se desea analizar desde un punto de vista conceptual, la aplicación de las herramientas Lean en las PYMES. Esto debido a que, las características particulares de las PYMES proveen fortalezas y debilidades respecto con la implantación de LM [12].

Para cumplir con el propósito del trabajo, se realizó una revisión de literatura basada en la sistematización de la información, la cual, permitió el análisis de la información e interpretación de las teorías asociadas al objeto de estudio. El documento está dividido de la siguiente forma: primero, se presenta la metodología empleada para realizar la revisión de literatura; seguidamente, los resultados de la investigación; en tercer lugar, las conclusiones; por último, se da a conocer la bibliografía consultada.

\section{METODOLOGÍA}

Se utilizaron motores de búsqueda como ScienceDirect, Proquest, Scielo y Scopus, además, se definieron palabras clave para la búsqueda como "LeanManufacturing", "5s", "Kaizen", "Heijunka", "Kanban", "SMED", "TPM", "JIDOKA", "ValueStreamMap", "Andon", "KPI's", "SixSigma", "PokaYoke", "Amfe". Se obtuvo un resultado inicial de 2.368.569 documentos. Para depurar la información se aplicaron tres filtros: i) estudiar los trabajos publicados en los últimos cinco años, ii) estudiar solo aquellos trabajos que son artículos producto de investigación, y iii) solo obtener investigaciones de aquellas revistas que abordan temas de manufactura, producción e ingeniería Industrial.

Con la aplicación del primer filtro, se obtuvo un resultado de 554.561 títulos. Tras la aplicación del segundo filtro la cifra cambió a 512.580. Aplicando el tercero, se redujo la cantidad a un total de 2.780 . Si bien el número de documentos disminuyó de 
forma considerable, fue necesario seguir filtrando hasta llegar a un número manejable para los autores. Se procedió a la lectura de los títulos de los artículos para seleccionar a aquellos considerados relevantes (artículos cuyo título mostrasen una intensión de aplicación de herramientas Lean). Resultado de ello, se obtuvo un total de noventa y dos (92) documentos considerados para realizar la lectura completa. Finalmente se seleccionaron cincuenta (50) artículos que evidenciaban en su contenido el estudio de alguna de las herramientas Lean.

\section{RESULTADOS Y ANÁLISIS}

A partir de la revisión de los conceptos relacionados con $L M$, se presentan los resultados en cuatro secciones, i) la tendencia en investigación sobre las herramientas Lean, ii) el análisis de herramientas Lean y su aplicación en la industria, iii) las variables de estudio durante la implantación de las herramientas Lean, y iv) las barreras de implementación de las herramientas Lean.

\subsection{Tendencia de la investigación}

En la Fig. 1, se puede visualizar el número de investigaciones realizadas sobre LM durante los últimos cinco (5) años.

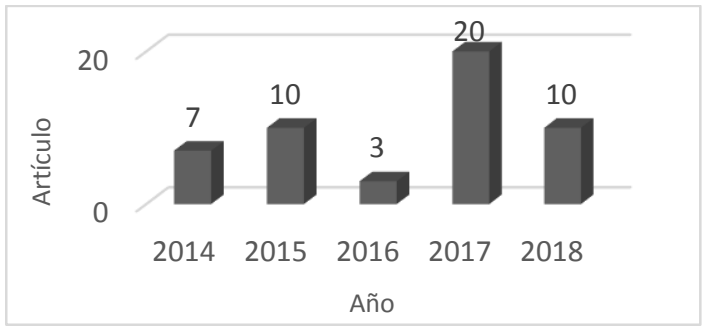

Fig. 1. Número de publicaciones por año

El número de artículos publicados sobre LM presentan un crecimiento en los últimos cinco años. Del año 2014 a 2015 el número de trabajos sobre el tema pasó de siete (7) a diez (10), y en el 2017se desarrollaron veinte (20) trabajos. Lo que muestra que el uso y aplicación de las herramientas Lean se ha tornado un factor importante en desarrollo de las industrias.

En el año 2016 el número de publicaciones disminuye, esto no quiere decir que no se hayan realizado aplicaciones de herramientas Lean, lo que denota es que en este año se publicaron menos trabajos sobre las aplicaciones de las herramientas Lean. En el año 2018 el número de publicaciones es menor respecto al año 2017, esto se debe a la ventana de tiempo en la que se realizó el estudio, la cual, fue hasta el primer semestre del 2018 (momento en que se realizó la revisión), por lo que, si se llegase a realizar la revisión de literatura hasta finalizar el año, este número publicaciones puede ser mayor, por ende, la tendencia de la investigación sobre el tema presentaría crecimiento mayor.

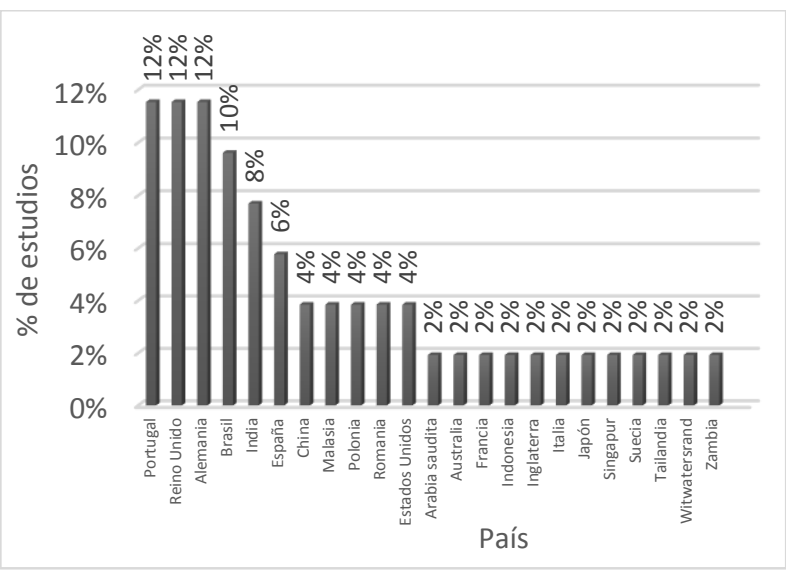

Fig. 2. Estudios por país

De acuerdo con la revisión de la literatura, se encontró que en los últimos cinco años se han desarrollado estudios sobre Lean en 24 países. En la Fig. 2, se ilustra el porcentaje de investigaciones que se han efectuado por país. En Portugal, el Reino Unido y Alemania se han desarrollado el $12 \%$ de las investigaciones respecto a Lean, respectivamente, siendo estos los países que más presentan avances en investigación; en Brasil se ha desarrollado el $10 \%$ de los estudios, siendo el cuarto país con más publicaciones sobre Lean; en la India se ha realizado el $8 \%$ y en España el 6\% de las investigaciones sobre el tema; en países como China, Malasia, Polonia, Rumania y Estados Unidos se ha realizado el $4 \%$ de los estudios, respectivamente; entre los países que menos desarrollan investigaciones sobre Lean, están Arabia saudita, Australia, Francia, Indonesia, Inglaterra, Italia, Japón, Singapur, Suecia, Tailandia, Witwatersrand y Zambia. Esto no quiere decir que los aportes en investigación no sean 
importantes, lo que muestra es que, en estos países existe menor interés en desarrollar estudios relacionados con la filosofía.

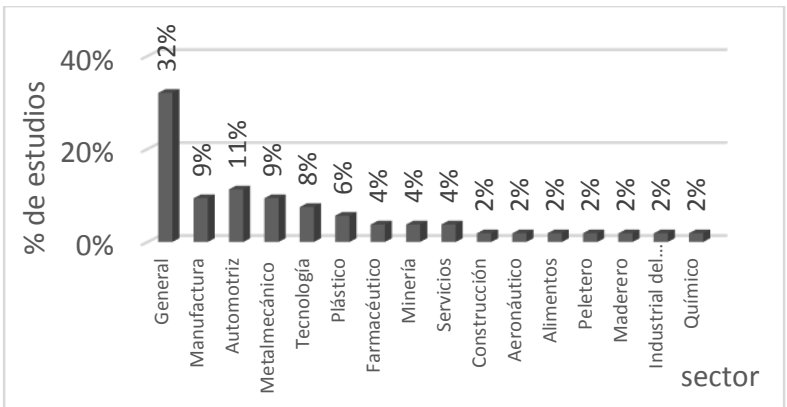

Fig. 3. Estudios por sector

Por otra parte, es importante tener en cuenta los sectores en los que se desarrollan las investigaciones respecto a Lean (ver Fig. 3); el 32\% de los autores consultados no indican el sector específico en los que desarrollaron el análisis, aunque muestran avances importantes respecto aLean; el 9\% de los autores manifiestan que los trabajos fueron desarrollados en empresas de manufactura sin especificar el tipo de producto que se procesa; el $11 \%$ de los autores desarrollan aplicaciones y estudios en la industria automotriz, siendo este el sector en el que más se aplica la filosofía Lean; el 9\% desarrollan investigaciones en la industria metalmecánica; el $8 \%$ estudia las empresas de tecnología; el 6\% realiza investigación en industrias del sector plástico; el 4\% realiza aplicaciones en los sectores de la minería, farmacéutico y de servicios, respectivamente. Los sectores en los que menos se desarrollan investigaciones respecto a Leanson el de la construcción, el aeronáutico, el alimenticio, el peletero, el maderero, la industria del color y la química, lo que representa un potencial para el desarrollo de lean en este tipo de empresas.

En la implementación de Lean no se debe introducir solo técnicas y métodos de gestión tradicionales, también se debe introducir ideas y sistemas de gestión innovadoras [13]. Existen autores que abordan los retos de la producción innovando las herramientas existentes, como en el caso de la industrial 4.0, considerada por algunos autores como la cuarta revolución industrial. En los estudios de[14], [5] y [15]se abordan los problemas de las fábricas de aprendizaje integrando herramientas Lean. En las fábricas de aprendizaje se enfrenta al desafío de trabajar con personal a tiempo parcial que no está familiarizado con grandes proyectos de tecnología y métodos de desarrollo [16].

En la fabricas de aprendizaje es efectivo impartir los principios y métodos Lean a los empleados en un corto tiempo. Todos los empleados pueden lograr una comprensión de Lean, independientemente de su punto de partida [15]. En el trabajo de [14], se estudia la aplicación de hardware de computadora de bajo costo y software de código abierto en áreas de mecanizado de fábricas de aprendizaje, empleando un sistema PokaYoke para la eliminación de errores en los procesos de fresado. Los resultados muestran que las fábricas de aprendizaje son eficaces para impartir habilidades Lean en un corto espacio de tiempo y que todos los empleados pueden lograr una comprensión de Lean.

En la investigación de [5] se analiza las competencias requeridas para lograr la integración entre LeanManagement y la Industria 4.0. Las competencias se pueden derivar y formular si los participantes entienden los principios de "Lean 4.0" (considerado como la integración entre LeanManufracturing e Industria 4.0) y son capaces de analizar el flujo de valor en el sistema. Al impartir principios y métodos Lean en las fábricas de aprendizaje se presenta un aumento en la comprensión del entrenamiento e incremento de habilidades [15].

Otros autoresestudian la aplicación de los robots colaborativos (CoBots) dentro de los sistemas productivos[17].Analizan flexibilidad de células de producción en "U" comparando la producción manual con la semiautomatizada con ayuda de un (CoBots). Para la producción en condiciones reales en células con forma de $U$ asistidas por CoBots, la productividad mejora[17], lo que sugiere que la robótica cooperativa se puede integrar en los sistemas de producción y aumentar la eficiencia.

En términos generales, existe una tendencia de aplicar Lean para la solución de problemas de fabricación en la industria moderna. Las herramientas Leanson adaptables a los requerimientos de las empresas, permiten la excelencia operacional a partir de la articulación de sus principios con las necesidades tecnológicas y de innovación de los sistemas de fabricación. 


\subsection{Aplicación de las herramientas Lean}

Se analiza la aplicación de catorce (14) herramientas consideradas esbeltas. En laFig. 4, se ilustran las herramientas y el número de veces que se han estudiado de acuerdo con las cincuenta (50) referencias consultadas.

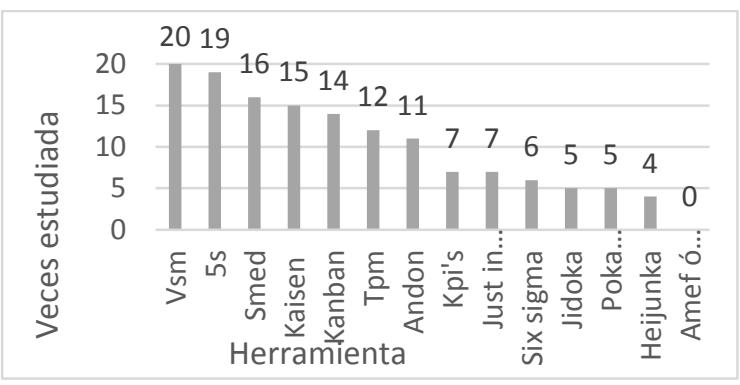

Fig. 4. Aplicación de las herramientas Lean

En la Fig. 4se puede ver que la herramienta preferida es el VSM con un total de veinte (20) aplicaciones. Seguidamente se encuentran herramientas como $5 s$, SMED, Kaizen y Kanban que son aplicadas para la mejora del orden, aseo, flexibilidad y la calidad. El TPM, el ANDON, los KPIs y el Just in Time presentan un total doce (12), once (11), siete(7) y siete(7) aplicaciones respectivamente. Herramientas como SixSigma, Jidoka, PokaYoke y Heijunka son las menos aplicadas, no por ello son consideradas irrelevantes, dado que los autores consultados evidencian su utilidad con los resultados que han obtenido. Si bien la herramienta de Amefno ha sido mencionada por los autores consultados, esto no quiere decir que la herramienta no sea útil para la solución de problemas bajo la filosofía Lean, lo que denota es que, durante los últimos cinco (5) años no se ha publicado sobre el uso de la herramienta.

Debido a que la herramienta de las $5 s$ es una de las más aplicadas en la literatura, es importante resaltar su excelencia para la mejora de los sistemas productivos, así como lo demuestran [19], quienes desarrollaron un modelo de dinámica de sistemas para la fabricación de envases de impresión, con el que estudiaron el rendimiento del sistema al aplicar la herramienta de las $5 s$. Evaluando el LeadTime, los autores muestran que existe un vínculo directo entre las mejoras en $5 s$ y el impacto en el rendimiento del sistema de fabricación [19].
El estudio de [20] desarrollado en el sector de la salud, buscó medir la satisfacción de las enfermeras aplicando el sistema Kanban durante la logística de los consumibles médicos. Se incluyó un análisis factorial exploratorio y un Análisis de Componentes Principales Categóricos (CAPTCHA). Como resultado se tiene que los encargados de la administración en hospitales deben promover la implementación de sistemas de Kanban, debido a que estos permiten el aumento de la satisfacción de los empleados [20].

Existen innovaciones en las herramientas Lean, adaptándolas a los requerimientos de la industria contemporánea. Ejemplo de esto es el trabajo de[21], quienes proponen una alternativa efectiva para la mejora del desempeño ecológico de las operaciones a partir del ciclo PHVA(Planear, hacer, verificar y actuar). [21] presentan el E-VSM (EnvironmentalValueStreamMapping), que permite abordar los problemas ambientales de las operaciones considerados críticos, esto contemplando los parámetros tradicionales del VSM.

Los modelos de gestión de operaciones se han desarrollado de acuerdo con los requerimientos de la demanda de la sociedad, tales como: producción limpia, productos reciclables, mejores condiciones de trabajo y la mejora de las condiciones sociales[22]. Con fines de lograr los objetivos económicos, sociales y ambientales dentro de la industria de la manufactura, [23] estudian la aplicabilidad del modelo propuesto por [24] denominado "SustainableValueStreamMapping (Sus-VSM)", considerado una extensión del VSMtradicional. De acuerdo con los resultados obtenidos por [23], se revela que se puede obtener información valiosa sobre el rendimiento de la sostenibilidad de los sistemas productivos basándose en el Sus-VSM sin importar la naturaleza del sistema.

En el trabajo de [25], se presenta una metodología para la integración de $L M$ con el ManufacturingExecutionSystems (MES) aplicada en el campo de la aeronáutica. La metodología permitió alos autores el diseño de un sistema para el posicionamiento de la pieza dentro de una máquina de fabricación, lo que se reflejó en mejor calidad para las piezas terminadas [25].

Durante las últimas décadas, la huella ambiental de la fabricación de productos se ha convertido en una 
preocupación de carácter público, lo que hace que las empresas revalúen los impactos ambientales de la fabricación [26]. En el trabajo de [27] se propone un modelo integrador entre $L M$ y producción más limpia (PML) denominado LCPC (Lean CleanerProduction Benchmarking), este busca la evaluación de la producción más limpia en empresas de diferentes tamaños. El modelo estudia los aspectos relacionados con la gestión de personas, información, productos, proveedores, clientes y procesos, así como las prácticas de $L M q u e$ favorecen a una producción ecoeficiente. Se evidencia que $L M$ puede ser integrado con otras herramientas relacionadas directamente con el control de la producción, lo que es favorable para la mejora de los sistemas productivos.

Si bien existe una correlación entre Lean y Green, las empresas han encontrado difícil integrar e implementar prácticas Lean y Green simultáneamente, especialmente cuando los recursos son limitados [28]. En el trabajo de[28] proponen una metodología para integrar las practicas Lean y Green, la cual, presenta una métrica denominada "CarbonValueEfficiency". La metodología fue probada en el sector metalmecánico, concluyendo que se puede mejorar la eficiencia de los tiempos de producción y de la huella del carbono tras su aplicación.

En el estudio de [9]se realiza una integración de Lean y Green, para ello proponen un nuevo modelo denominado "Modelo Lean \& Green". El cual tiene como objetivo la disminución del impacto ambiental e incremento de la productividad. El modelo adopta un enfoque Kaizen para la mejora de los flujos en el sistema de masa y energía en los entornos de fabricación [9].

Los autores[29] estudian el EVSM (EnergyValueStreamMapping) para abordar los procesos que consumen energía no productiva centrándose en una fabricación ecológica, de lo que establecen que, con el diseño del mapeo de flujos de valor de energía (EVSM) se puede desarrollar un sistema de fabricación esbelto y ecológico.

En el trabajo de [30] se estudian más de treinta (30) herramientas consideradas Lean para regular el entorno de la producción en noventa y un (91) empresas del sector automotriz. Se basaron en una encuesta aplicada a las empresas participantes y el método de promedio ponderado para el análisis de las herramientas de mayor impacto en el sector. Producto de la investigación, se obtiene que hay cinco herramientas consideradas altamente efectivas para regular los sistemas productivos (las $5 s$, la eficacia general del equipo, la metodología de solución de ocho pasos, el análisis de Pareto y la eliminación de residuos), de las que se destacan la herramienta de las 5 s como una de las de mayor impacto[30].

En el trabajo de [18] se presenta un Modelo de Servicio Lean, que tiene en cuenta el papel del cliente en la creación del servicio. El modelo se presentó en un ciclo conceptual y uno práctico. El ciclo conceptual describe los principales principios y desperdicios de la filosofía Lean, asegurando su aplicabilidad en las actividades de servicio. El ciclo práctico es una guía para la introducción de Lean en las empresas de servicios. Dicho sector puede beneficiarse con la filosofía Lean a través del aumento de la competitividad organizacional, la satisfacción del cliente y la reducción desperdicios del proceso [18].

En virtud a lo anteriormente descrito, las herramientas Lean se pueden aplicar en todo tipo de organizaciones debido a su adaptabilidad para solucionar problemas. En la literatura se evidencia la aplicabilidad de herramientas en empresas de automoción, de la industria gráfica e inclusive empresas de servicios como la salud. Adicional a ello, es posible integrar herramientas Lean (como Kaizen, 5s, VSM entre otras), con herramientas para el control de la producción y/o de la calidad (como PML, Green, Pareto y los ciclos de calidad). Diversidad de autores buscan adaptar las herramientas Lean a las necesidades de los sistemas productivos contemporáneos, esto evaluando la sostenibilidad desde los enfoques tradiciones de reducción de desperdicio, así como impactos ambientales y sociales.

\subsection{Variables estudiadas durante la implantación de LeanManufacturing}

En la Fig. 5se puede visualizar las variables que se han estudiado durante la aplicación de las herramientas Lean. Se han ordenado de forma descendente de acuerdo con el número veces que se han estudiado por los diferentes autores. 


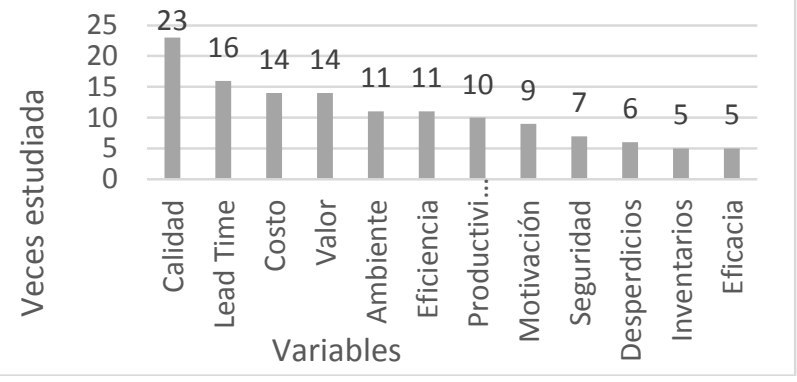

Fig. 5. Variables estudiadas

De acuerdo con la Fig. 5, se puede establecer que las variables tradiciones de estudio dentro del modelo Toyota (Calidad, Costos, Tiempos de entrega, seguridad y motivación) no son las únicas estudiadas, muchos autores miden la aplicación de las herramientas empleando variables diferentes como el valor, el impacto medio ambiental, la eficiencia la productividad, entre otras.

La calidad es la variable que más se ha estudiado, contando con un total de veinte y tres (23) veces que se ha involucrado en la mejora de los sistemas de fabricación. El LeadTime ha sido estudiado dieciséis (16) veces, esto buscando la mejora de los tiempos de respuesta aplicando principalmente la herramienta SMED. Los costos y el valor se han estudiado catorce (14) veces, esto empleando principalmente el VSM, las 5s y el Kaizen. La variable de medio ambiente se ha estudiado por once (11) autores que buscan disminuir impactos ambientales. La eficiencia y la eficacia cuentan con once (11) y cinco (5) aplicaciones respectivamente, estas variables se tienden a medir empleando $\mathrm{KP} / \mathrm{s}$, y en algunos casos para ver el efecto de TPM o VSM en los sistemas de fabricación. La variable de productividad se ha aplicado diez (10) veces, esta variable se emplea por los autores para la medición de los resultados tras la aplicación de herramientas como Kaizen, 5s, SMED, TPM, ANDONóVSM. La motivación se ha estudiado nueve (9) veces, principalmente aplicando herramientas como KaizenSMED y ANDON. Los desperdicios se han estudiado siete (7) veces, se tienden a medir aplicando herramientas como Kaizen, 5s y SixSigma. En cinco (5) casos se ha estudiado los inventarios dentro de los sistemas productivos, esto aplicando principalmente la herramienta SMED y Kaizen.

El mantenimiento comparte costos operativos significativos en una organización y es considerado como un pilar principal del desempeño organizacional [31]. Es necesario analizar los planes de mantenimiento para la mejora de los sistemas productivos en las empresas. En el estudio de [32] sobre el mantenimiento y control de la maquinaria en líneas de producción, se realiza un análisis de indicadores clave de rendimiento $(K P I)$ y el impacto sobre el tiempo de actividad de una línea de montaje y en la inspección de calidad. Muestran la utilidad los KPls para medir variables consideradas fundamentales como la calidad y la efectividad general del equipo (OEE).

El sector automotriz constituye una de las actividades más exigentes del mercado global, ya que requiere un aumento constante de la productividad [33] y aprovechamiento de la maquinaria y equipos [20]. Los autores [20] realizan un estudio en este sector, buscan el aumento de la disponibilidad de la maquinaria y equipo a través de la implementación de un plan de mantenimiento. Aplicaron el TPM y algunas prácticas de gestión visual (ANDON), lo que permitió medir la eficiencia global del equipo (OEE). Entre otros hallazgos obtenidos por [20], se tiene que, al aplicar estas herramientas, no solo se mejora el $O E E$, sino que también se desarrolla en los operadores la responsabilidad de contribuir con la limpieza, organización y verificación de puntos críticos en cada estación de trabajo, lo cual asegura que las maquinas estén en funcionamiento todo el tiempo.

El estudio de herramientas como SixSigma se ha convertido en un factor de interés para la mejora continua de los sistemas productivos, ya que contribuye a reducir los costos de la no calidad mediante la búsqueda de "cero defectos" [34]. En el trabajo de [35], se identificó la correlación entre LeanSixSigma y la sostenibilidad organizacional. Los autores identificaron cinco (5) impactos influyentes sobre la organización y la importancia de la dimensión del costo para la sostenibilidad en las organizaciones. Los impactos de LeanSixSigma son (i) la reducción del desperdicio, (ii) la reducción de la tasa de defectos, (iii) la mejora de la calidad del servicio, (iv) el aumento de la calidad del producto y (v) la reducción de la variabilidad del proceso. Los dos primeros están relacionados con la dimensión costos y los otros tres están alineados con la dimensión Calidad [35].

El modelo de las $5 s$ es considerado por algunos autores como el modelo de gestión más utilizado en la industria automotriz. Estees estudiado en el 
trabajo de[36], donde se analiza la relación entre la evolución de las $5 \mathrm{~s}$ y la productividad en una empresa del sector automotriz en Rumania. El autor establece que existe una correcta relación positiva entre las $5 s$ y la productividad, lo que significa que implantar las $5 s$ en la empresa conduce a mejores resultados.

En el trabajo de [21]se aplican gran número de herramientas Lean en la industria del cuero y la madera, entre estas Kaizen, 5s, SMED, Kanban, TPM, VSM y JustinTime, para la mejora de la calidad, la flexibilidad, el impacto medio ambiental y la seguridad. [21]presentan los resultados de su investigación como una novedad debido a que, consideran la utilización de las herramientas Lean en el rendimiento de las medidas ambientales, lo cual, es de utilidad para los gerentes que desean aplicar la filosofía Lean y a su vez cumplir con reglamentaciones ambientales.

En el estudio de [11] se investiga el impacto de cinco métodos Lean (JIT, Jidoka, Kaizen, TPM y VSM) para el cumplimiento del desempeño ambiental medido en términos de uso de material, consumo de energía, producción no productiva y emisiones de contaminantes. El estudio fue realizado con doscientas cincuenta (250) organizaciones de fabricación de todo el mundo, y se obtuvo que, herramientas como TPM y JIT presentan una mayor relevancia para la mejora del desempeño ambiental. [11] presentan una visión interesante sobre los efectos de la fabricación ajustada en el desempeño ambiental, lo que puede permitir a las organizaciones que no están comprometidas 0 que están parcialmente comprometidas con la sostenibilidad obtener beneficios a sus operaciones.

Adicionalmente, en el trabajo de [37] se propone un modelo para evaluar algunos de los pilares de Lean con el cumplimiento de los requisitos ambientales contemporáneos. Se evaluó el Just in Time, el Jidoka y respeto por las personas (RfP) desde una perspectiva de taller. Se obtuvo que el Jidoka y el RfP tienen un impacto positivo en el desempeño ambiental, mientras que las herramientas $y$ prácticas de Just in Time tienen un impacto negativo.

El estudio de la flexibilidad y el Lead Time se presenta en el trabajo de [38], donde se hizo un estudio de caso a una empresa del sector automotriz especializada en la fabricación de cables de comando para automóviles (aquellos que liberan puertas, ventanas, asientos, etc.). Debido a la diversidad de referencias se requería la reducción de los tiempos de preparación, para lo que se implementó la técnica SMED complementada con otras herramientas como $5 s$ y Andon. Como resultado se obtuvo una reducción de los tiempos de configuración reflejado en el aumento de la flexibilidad y la productividad [38].

La ergonomía del trabajo es un factor importante para estudiar dentro de las condiciones de seguridad de los sistemas productivos. Es esencial para mejorar estaciones de trabajo y beneficiar la correlación entre los empleados de la empresa [39]. En el trabajo de [40] se hizo un estudio en el sector metalúrgico sobre las condiciones de seguridad, específicamente, se evaluó la ergonomía de los puestos de trabajo. Igualmente, se estudiaron los tiempos en la configuración de la maquinaria, debido a que se presentaban problemas de productividad. Para remediar los problemas en el sistema productivo, [40]integraron el estudio de las condiciones ergonómicas junto con la aplicación de la herramienta SMED, Andon y las 5s.

En el trabajo de[41] se estableció que el TPM se puede utilizar como una herramienta para la mejora de la eficiencia general de los equipos (OEE), esto debido a permite la reducción de pérdidas y el retrabajo en los sistemas productivos a niveles aceptables, y al mismo tiempo ayudar a las empresas a incrementar la rentabilidad garantizando la competitividad ante otras empresas.

Las variables tradiciones estudiadas en el modelo de producción Toyota (Calidad, Costos, Tiempos de entrega, Seguridad y Motivación)mantienen una importancia significativa para las empresas contemporáneas. Se busca controlar su comportamiento manteniéndolas en márgenes a aceptables; sin embargo, diversidad de autores centran su atención en el estudio de otras variables como el valor, la sostenibilidad ambiental y social, esto a partir del uso y adaptación de las herramientas Lean a las necesidades que presentan los sistemas de fabricación.

\subsection{Barreras para la implantación de LPS en las PYMES}

Muchas empresas con el fin de reducir los costos, utilizan materiales de baja calidad o sobrecargan a 
los empleados; no se dan cuenta de las oportunidades para mejorar los sistemas productivos y están preocupadas por lo desconocido, lo cual, es un problema particular de los muchos relacionados con LM[42].

Para realizar una implementación exitosa del LeanProductionSystem (LPS) se necesita integrar e implementar simultáneamente los elementos Lean[43]. Sin embargo, las PYMES a menudo carecen del conocimiento para desarrollar LPS[44], por lo que, es necesario conocer las barreras de las PYMES para poder implantar LPS. Se requiere integrar estrategias parafortalecer los sistemas productivos [45], esto con el fin de superar las barreras que se puedan presentar durante el estudio y aplicación de las herramientas.

El conflicto entre las características de las PYMES y la implementación deLeanradica en la falta de recursos, la falta de experiencia, la falta de procedimientos y métodos, la estrategia a corto plazo y la organización no funcional [11]. Para la toma de decisiones se centra la responsabilidad en una sola persona, que usualmente es el dueño de la empresa y existe una falta de delegación impide el surgimiento de nuevos líderes [11]. Los líderes de la PYMESgarantizan un alto nivel de interacción con los sistemas productivos, dado que están altamente involucrados en el funcionamiento de sus sistemas productivos [11]. Esto último es considerado como una ventaja para las PYMES al momento de implantar la metodología Lean.

La intervención de la alta dirección en la implementación de Lean Manufacturinges un factor clave de éxito, especialmente para las PYMES [46]. La alta dirección debe mostrar una fuerte intención de la aplicación de las herramientas Lean, de lo contrario, los esfuerzos realizados por la parte operativa serían insuficientes para la mejora de los sistemas productivos [46]. En el trabajo de [44] se describen diecisiete (17) factores de éxito y diecisiete (17) barreras existentes para la implantación de $L P S$ en las PYMES delsector farmacéutico. Entre las barreras descritas por [44], se resaltan tres, la falta de compromiso de la gestión, la falta de conocimiento y la resistencia a Lean. Si se desea, aplicar el modelo de LPS en las PYMES, se recomienda tener en cuenta las barreras existentes durante la implantación [44]. Autores como [47] clasifican las barreras para la implementación de $L M$ en barreras financieras, barreras de gestión, barreras de la fuerza de trabajo y otras barreras (Distracciones por la existencia de múltiples proyectos).

Para la implantación de $L M$ en la PYMES, es necesario estudiar la cultura organizacional. Se debe estudiar la satisfacción de los empleados, dado que puede ser una barrera importante que tener en cuenta durante la mejora de los sistemas productivos. Además, es preciso analizar aspectos relacionados con la comunicación e interacción en la organización, la estrategia y la visión [48] buscando un horizonte claro de lo que se desea con la implantación de Lean.

Otros autores estudian como tal la implementación simultánea de LeanManufacturing y SixSigma, de los que se puede decir que, para la implantación paralela de estas herramientas en las empresas, principalmente en las PYMES, la falta de los recursos necesarios es una barrera que se debe superar [49]. Por lo que es importante el estudio de la capacidad de los recursos que poseen las PYMES para la integración de las herramientas.

En la investigación de [14] se estudia acerca de una posible aplicación de hardware de computadora de bajo costo y software de código abierto en áreas de mecanizado en fábricas de aprendizaje. Los autores demuestran la capacidad de los componentes de hardware de bajo costo para cumplir con los requisitos de la fabricación moderna [14], siendo este un claro ejemplo de implementación de herramientas Lean de bajo costo. Los volúmenes de información en un sistema de control de producción pueden dificultar la toma de decisiones, por lo que, para tomar las decisiones correctas se requieren de la información precisa [50]. La calidad de la información se torna un factor importante para el estudio de los sistemas productivos, debido a que, con la información adecuada en el momento adecuado, las empresas pueden decidir cuándo actuar y cómo actuar para ser más competitivos.

En el estudio de [51] abordan un aspecto poco abordado por la literatura de Lean-TI, de lo que se establece que "la solución técnica de problemas depende especialmente de la confiabilidad de los datos, el flujo de información en tiempo real y la gestión de conflictos en la integración del conocimiento" [51]. Es vital la presencia de sistemas de información para la implantación de herramientas Lean, especialmente cuando se integran tecnología de la información. 
Se debe estudiar detalladamente las herramientas Lean que requieran menor inversión y que a su vez resulten prácticas para la mejora de los sistemas productivos de las PYMES. Es conveniente emplear herramientas que no requieran grandes esfuerzos económicos para su implantación, como 5 s, PokaYoke, SMED, Kanban entre otras. Por otra parte, debe existir un alto compromiso en toda la organización para aplicar Lean, tanto en el personal operativo como en la alta dirección, sin el compromiso total del personal, los esfuerzos particulares no aportarían resultados fructuosos a las PYMES. Adicionalmente, si existe compromiso por parte del personal de la empresa, es más fácil superar otras barrares como la falta de conocimiento en la aplicación de Lean.

\section{CONCLUSIONES}

Las herramientas Lean más aplicadas para la mejora de los sistemas productivos son el ValueStreamMapping, las $5 \mathrm{~s}$, el SMED y el Kaizen, lo cual, muestra que los principales problemas que se abordan en la industria están asociados con análisis de valor, el orden y aseo, y la flexibilidad de los sistemas productivos. Esto sin subvalorar la importancia de las demás herramientas para la mejora de otras variables importantes en la mejora de los sistemas productivos.

Las herramientas Leanson adaptables a las necesidades de las empresas, sin importar si son empresas de manufactura 0 de servicios. Diversidad de autores buscan integrarLean con otras herramientas de gestión de producción y control de la calidad (como PML, Green, Análisis de Pareto, ciclos de calidad entre otros), para la solución de problemas de fabricación contemporáneos. Lo que evidencia la necesaria evolución de las herramientas Lean para abordar las necesidades de las empresas contemporáneas.

Las variables tradiciones de estudio dentro del modelo Toyota (Calidad, Costos, Tiempos de entrega, seguridad y motivación) aún son abordadas para analizar los sistemas productivos; sin embargo, muchos autores optan por medir el rendimiento de los sistemas evaluando variables diferentes como el valor, el impacto medio ambiental, la eficiencia la productividad, entre otras variables. El impacto ambiental y el valor son variables que se han estudiado con detalle en la literatura, lo que evidencia que, las herramientas Lean se están ajustando a los requerimientos de la industria contemporánea. Es necesario que las investigaciones sobre las herramientas Lean se hagan en pro de solucionar los requerimientos de la industria contemporánea y no limitarse solamente a los modelos tradicionales.

Durante los últimos cinco años no se evidencia la aplicación de la herramienta de Amfeo Amef para la mejora de los sistemas productivos. Esto no quiere decir que la herramienta no sea útil. Para aquellos investigadores que deseen profundizar el conocimiento respecto a la utilización de esta herramienta, se recomienda consultar la literatura en periodos anteriores al 2014, dado que la ventana de tiempo del presente estudio se manejó en un periodo de cinco años, desde el 2014 al 2018.

En términos generales, las barreras para la implantación de Lean en las PYMES, se pueden resumir en tres i) el compromiso institucional, ii) el conocimiento sobre las herramientas Leany iii) los recursos económicos necesarios para la puesta en marcha. Todas estas barreras se pueden superar; sin embargo, es necesario analizar cómo hacerlo. La primera es vital para la correcta permeabilización de la filosofía en los sistemas de fabricación, ya que debe existir un alto grado de compromiso de todo el personal, principalmente de quienes dirigen los procesos al interior de las empresas. Para superar la segunda barrera, existe literatura rica en información sobre cómo aplicar la filosofía, lo que facilita el acceso al conocimiento sobre Lean, simplemente se requiere compromiso y motivación para aplicar la filosofía. En cuanto a los recursos económicos, las PYMES presentan menor capacidad de inversión que las grandes empresas, por lo que es conveniente iniciar con la aplicación de aquellas herramientas que no requiera mayor inversión (5s, el PokaYoke, SMED yKanban).

\section{AGRADECIMIENTOS}

A la Universidad Militar Nueva Granada por la financiación del Proyecto INV-ING 2615 "Propuesta metodológica para la aplicación de herramientas del Sistema de Producción Toyota en las Pymes textileras ubicadas en el Departamento de Cundinamarca" del cual, el presente artículo es resultado de investigación. 


\section{REFERENCIAS BIBLIOGRÁFICAS}

[1] A.-A. Karam, M. Liviu, C. Veres y H. Radu, «The contribution of lean manufacturing tools to changeovertime decrease in the pharmaceutical industry. A SMED project,» Procedia Manufacturing, vol. 22, pp. 886-892, 2018.

[2] S. Indrawati y M. Ridwansyah, «Manufacturing Continuous Improvement Using Lean Six Sigma: An Iron Ores Industry Case Application,» Procedia Manufacturing, vol. 4, p. 528 - 534, 2015.

[3] J. Choomlucksana, . M. Ongsaranakorn y P. Suksabai, «Improving the productivity of sheet metal stamping subassembly area using the application of lean manufacturing principles,» Procedia Manufacturing, vol. 2, pp. 102-107, 2015.

[4] A. Azizi y T. Manoharan, «Designing a Future Value Stream Mapping to Reduce Lead Time using SMED-A Case Study," Procedia Manufacturing, vol. 2, pp. 153-158, 2015.

[5] J. Enke, R. Glass, A. Kreß, J. Hambach, . M. Tisch y J. Metternich, «Industrie 4.0 Competencies for a modern production system,» Procedia Manfacturing, vol. 23, pp. 267-272, 2018.

[6] J. Mohd Rohani y S. Mojib Zahraee, «Production line analysis via value stream mapping: a lean manufacturing process of color industry,» Procedia Manufacturing, vol. 2, p. 6 $-10,2015$.

[7] T. Ohno, El sistema de producción Toyota: Más allá de la producción a escala, Barcelona: Ediciones Gestión 2000, S.A., 1991.

[8] M. Kumar, R. Vaishya y Parag, «REAL-TIME MONITORING SYSTEM TO LEAN MANUFACTURING, Procedia Manufacturing, vol. 20, pp. 125-140, 2018.

[9] A. Brasco Pampanelli, P. Found y A. Moura Bernardes, «A Lean \& Green Model for a production cell, » Journal of Cleaner Production, pp. 1-12, 2017.
[10] J. Oliveira, J. Sá y A. Fernandes, «Continuous improvement through "Lean Tools": An application in a mechanical company,» Procedia Manufacturing, vol. 13, pp. 10821089, 2017.

[11] J. A. Garza Reyes, . V. Kumar, S. Chaikittisilp y K. Hua Tan, «The effect of lean methods and tools on the environmental performance of manufacturing organisations,» International Journal of Production Economics, 2018.

[12] A. Moeuf, S. Tamayo, S. Lamouri, R. Pellerin y A. Lelievre, "Strengths and weaknesses of small and medium sized enterprises regarding the implementation of lean manufacturing," IFAC-PapersOnLine, vol. 49, no 12, p. 071076, 2016.

[13] M. K. Wyrwicka y B. Mrugalska, «Mirages of Lean Manufacturing in Practice,» Procedia Engineering, vol. 182, p. $780-785,2017$.

[14] M. Wiech, J. Böllhoff y J. Metternich, «Development of an optical object detection solution for defect prevention in a Learning Factory,» Procedia Manufacturing, vol. 9, pp. 190-197, 2017.

[15] S. Makumbe, T. Hattingh, N. Plint y D. Esterhuizen, «Effectiveness of using Learning Factories to impart Lean principles in mining employees,» Procedia Manufacturing, vol. 23, pp. 69-74, 2018.

[16] C. Hofmann, S. Lauber, B. Haefner y G. Lanza, «Development of an agile development method based on Kanban for distributed part-time teams and an introduction framework, , Procedia Manufacturing, vol. 23, pp. 45-50, 2018.

[17] F. Gil Vilda, A. Sune, J. A. Yagüe Fabra, C. Crespo y $\mathrm{H}$. Serrano, «Integration of a collaborative robot in a U-shaped production line: a real case study,» Procedia Manufacturing, vol. 13, pp. 109-115, 2017.

[18] A. López, I. González Requena y A. Sanz Lobera, "Lean Service: Reassessment of Lean Manufacturing for Service Activities,» Procedia 
Engineering, vol. 132, p. 23 - 30, 2015.

[19] O. Omogbai y K. Salonitis, «The implementation of $5 \mathrm{~S}$ lean tool using system dynamics approach,» Procedia CIRP, vol. 60, p. $380-385,2017$.

[20] V. G. Aguilar Escobar, S. Bourque y N. Godino Gallego, «Hospital kanban system implementation: Evaluating satisfactionof nursing personnel,» Investigaciones Europeas de Dirección y Economía de la Empresa, vol. 21, pp. 101-110, 2015.

[21] J. A. Garza Reyes, J. Torres Romero, K. Govindan, A. Cherrafi y U. Ramanathan, «A PDCA-based approach to Environmental Value Stream Mapping (EVSM), , Journal of Cleaner Production, vol. 180, pp. 235-248, 2018.

[22] A. L. Helleno, A. J. Isaias de Moraes y A. Tadeu Simon, «Integrating sustainability indicators and Lean Manufacturing to assess manufacturing processes: Application case studies in Brazilian industry,» Journal of Cleaner Production, vol. 153, pp. 405-416, 2017.

[23] A. Brown, J. Amundson y F. Badurdeen, «Sustainable value stream mapping (Sus-VSM) in different manufacturing system configurations: application case studies,» Journal of Cleaner Production, vol. 85, p. 164e179, 2014.

[24] W. Faulkner y F. Badurdeen, «Sustainable Value Stream Mapping (Sus-VSM): methodology to visualize and assess manufacturing sustainability performance,» Journal of Cleaner Production, pp. 1-11, 2014.

[25] D. A. Gianluca, J. Sauza Bedolla y P. Chiabert, «A novel methodology to integrate Manufacturing Execution Systems with the lean manufacturing approach,» Procedia Manufacturing, vol. 11, p. 2243 - 2251, 2017.

[26] W. M. Cheung, J. T. Leong y P. Vichare, «Incorporating lean thinking and life cycle assessment to reduce environmental impacts of plastic injection moulded products, 》 Journal of Cleaner Production, vol. 167, pp. 759-775,
2017.

[27] A. Ribeiro Ramos, J. C. Espíndola Ferreira, V. Kumar y J. A. Garza Reyes, "A lean and cleaner production benchmarking method for sustainability assessment: A study of manufacturing companies in Brazil, » Journal of Cleaner Production, vol. 177, pp. 218-231, 2018.

[28] R. Ng, J. S. Choong Low y B. Song, «Integrating and Implementing Lean and Green Practices based on Proposition of CarbonValue Efficiency Metric,» Journal of Cleaner Production, 2015.

[29] N. Verma y V. Sharma, «Energy Value Stream Mapping a Tool to develop Green Manufacturing,» Procedia Engineering, vol. 149, p. 526 - 534, 2016.

[30] P. Arunagiri y A. Gnanavelbabu, «Identification of High Impact Lean Production Tools in Automobile Industries using Weighted Average Method,» Procedia Engineering, vol. 97, p. 2072 - 2080, 2014.

[31] S. Mostafa, J. Dumrak y H. Soltan, «Lean maintenance roadmap,» Procedia Manufacturing, vol. 2, p. 434 - 444, 2015.

[32] N. Stricker, M. Micali, D. Dornfeld y G. Lanza, "Considering Interdependencies of KPls Possible Resource Efficiency and Effectiveness Improvements,» Procedia Manufacturing, vol. 8, p. $300-307,2017$.

[33] P. Guariente, I. Antoniolli, L. Pinto Ferreira, T. Pereira y F. Silva, «Implementing autonomous maintenance in an automotive components manufacturer,» Procedia Manufacturing, vol. 13, p. 1128-1134, 2017.

[34] S. Chen, S. Fan , J. Xiong y W. Zhang, «The Design of JMP/SAP Based Six Sigma Management System and its Application in SMED, » Procedia Engineering, vol. 174, p. 416 - 424, 2017.

[35] J. Galdino de Freitas, H. Gomes Costa y F. Toledo Ferraz, «Impacts of Lean Six Sigma over organizational sustainability: A survey 
Study,» Journal of Cleaner Production, vol. 156, pp. 262-275, 2017.

[36] C. Veres, . L. Marian, S. Moica y K. Al-Akel, "Case study concerning $5 S$ method impact in an automotive company,» Procedia Manufacturing, vol. 22, p. 900-905, 2017.

[37] A. Sartal , A. I. Martinez Senra y V. Cruz Machado , "Are all Lean Principles Equally Eco-Friendly? A Panel Data Study,» Journal of Cleaner Production, 2017.

[38] C. Rosa, F. Silva, L. Pinto Ferreira y R. Campilho, «SMED methodology: The reduction of setup times for Steel Wire-Rope assembly lines in the automotive industry,» Procedia Manufacturing, vol. 13, pp. 1034-1042, 2017.

[39] Z. G. Dos Santos, L. Vieira y G. Balbinotti, «Lean Manufacturing and ergonomic working conditions in the automotive industry,» Procedia Manufacturing, vol. 3, p. 5947 - 5954, 2015.

[40] M. Brito, A. Ramos, P. Carneiro y M. Gonçalves, "Combining SMED methodology and ergonomics for reduction of setup in a turning production area,» Procedia Manufacturing, vol. 13, p. 1112-1119, 2017.

[41] B. G. Mwanza y C. Mbohwa, «Design of a total productive maintenance model for effective implementation: Case study of a chemical manufacturing company,» Procedia Manufacturing, p. 461 - 470, 2015.

[42] K. Antosz y D. Stadnicka, «Lean Philosophy Implementation in SMEs - Study Results, , Procedia Engineering, vol. 182, p. 25 - 32, 2017.

[43] R. Sundar, A. Balaji y R. Satheesh Kumar, «A Review on Lean Manufacturing Implementation Techniques,» Procedia Engineering, vol. 97, p. $1875-1885,2014$.

[44] F. Sieckmann, H. Nguyen Ngoc, . R. Helm y . $\mathrm{H}$. Kohl, «Implementation of lean production systems in small and mediumsized pharmaceutical enterprises,» Procedia
Manufacturing, vol. 21, pp. 814-821, 2018.

[45] M. Kurdve, M. Zackrisson, M. Wiktorsson y U. Harlin, «Lean and green integration into production system models - experiences from Swedish industry," Journal of Cleaner Production, pp. 1-11, 2014.

[46] M. Alefari, K. Salonitis y Y. Xu , «The role of leadership in implementing lean manufacturing,» Procedia CIRP, vol. 63, p. 756 - 761, 2017.

[47] K. Salonitis y C. Tsinopoulos, "Drivers and Barriers of Lean Implementation in the Greek Manufacturing Sector,» Procedia CIRP, vol. 57, pp. 289-194, 2016.

[48] A. Alkhoraif y P. McLaughlin, «Lean implementation within manufacturing SMEs in Saudi Arabia: Organizational culture aspects, » Journal of King Saud University - Engineering Sciences, pp. 1-11, 2018.

[49] I. Alhuraish, C. Robledo y A. Kobi, «A Comparative Exploration of Lean Manufacturing and Six Sigma in terms of their Critical Success Factors,» Journal of Cleaner Production, 2017.

[50] Q. Gong, Y. Yang y S. Wang, «Information and decision-making delays in MRP, KANBAN, and CONWIP,» Int. J. Production Economics, vol. 156, pp. 208-213, 2014.

[51] A. Sartal y X. Vázquez, Journal of Manufacturing Systems, Vol 1 de Implementing Information Technologies and Operational Excellence: Planning, emergence and randomness in the survival of adaptivemanufacturing systems, 45, pp. 1-16, 2017. 